\title{
Childhood atopic dermatitis: A cross-sectional study of relationships between child and parent factors, atopic dermatitis management, and disease severity
}

\section{Authors:}

Corresponding author:

Amy E. Mitchell, PhD, RN, Research Coordinator, Parenting and Family Support Centre, The University of Queensland, Brisbane, Australia.

Tel.: +61 733461202

Fax: +61 733656724

Email: a.mitchell5@uq.edu.au

Jennifer A. Fraser, PhD, RN, Director (Research Students and Research Development), Sydney Nursing School, University of Sydney, Sydney, Australia.

Joanne Ramsbotham, PhD, RN, Lecturer, School of Nursing and Midwifery, Queensland University of Technology, Brisbane, Australia.

Alina Morawska, PhD, Deputy Director (Research), Parenting and Family Support Centre, The University of Queensland, Brisbane, Australia.

Patsy Yates, PhD, RN, Professor, School of Nursing and Midwifery, Queensland University of Technology, Brisbane, Australia.

\section{Note:}

At the time this research was conducted, Amy E. Mitchell was at the School of Nursing and Midwifery, Queensland University of Technology, Brisbane, Australia.

\section{ABSTRACT}

Background: Successful management of atopic dermatitis poses a significant and ongoing challenge to parents of affected children. Despite frequent reports of child behaviour 
problems and parenting difficulties, there is a paucity of literature examining relationships between child behaviour and parents' confidence and competence with treatment.

Objectives: To examine relationships between child, parent, and family variables, parents' self-efficacy for managing atopic dermatitis, self-reported performance of management tasks, observed competence with providing treatment, and atopic dermatitis severity.

Design: Cross-sectional study design.

Participants: A sample of 64 parent-child dyads was recruited from the dermatology clinic of a paediatric tertiary referral hospital in Brisbane, Australia.

Methods: Parents completed self-report questionnaires examining child behaviour, parents' adjustment, parenting conflict, parents' relationship satisfaction, and parents' self-efficacy and self-reported performance of key management tasks. Severity of atopic dermatitis was assessed using the SCORAD (Scoring Atopic Dermatitis) index. A routine home treatment session was observed, and parents' competence in carrying out the child's treatment assessed. Results: Pearson's and Spearman's correlations identified significant relationships $(p<.05)$ between parents' self-efficacy and disease severity, child behaviour difficulties, parent depression and stress, parenting conflict, and relationship satisfaction. There were also significant relationships between each of these variables and parents' self-reported performance of management tasks. More profound child behaviour difficulties were associated with more severe atopic dermatitis and greater parent stress. Using multiple linear regressions, significant proportions of variation in parents' self-efficacy and self-reported task performance were explained by child behaviour difficulties and parents' formal education. Self-efficacy emerged as a likely mediator for relationships between both child behaviour and parents' education, and self-reported task performance. Direct observation of treatment sessions revealed strong relationships between parents' treatment competence and parents' self-efficacy, outcome expectations, and self-reported task performance. Less 
competent task performance was also associated with greater parent-reported child behaviour difficulties, parent depression and stress, parenting conflict, and relationship dissatisfaction.

Conclusion: This study revealed the importance of child behaviour to parents' confidence and practices in the context of atopic dermatitis management. Children with more severe atopic dermatitis are at risk of presenting with challenging behaviour problems and their parents struggle to manage the condition successfully.

\section{Keywords}

Child behaviour; chronic disease management; cross-sectional studies; dermatitis, atopic; eczema; health behaviour; outcome expectations; parenting; self-efficacy. 


\section{INTRODUCTION}

Although atopic dermatitis (AD) has long been recognised as a common health problem among children (Williams et al., 1999), recent international studies suggest the problem is worsening, with prevalence increasing steadily over past decades in Western Europe, Canada, South America, Australasia, and the Far East (Williams et al., 2008). Children commonly develop AD at an early age, most within the first year of life (Ben-Gashir et al., 2004, Kay et al., 1994), and although many "grow out" of AD over time, almost half (47\%) of those with $\mathrm{AD}$ at 7 years of age continue to experience symptoms until age 11 , and one third $(35 \%)$ have AD persisting into adulthood (Williams and Strachan, 1998).

The impact of AD on the physical, psychological, and social wellbeing of affected children, their parents, and family, is substantial (Lewis-Jones, 2006). Treatment can be timeconsuming, complex and costly (Su et al., 1997), and management poses a significant and ongoing challenge for many parents (Zuberbier et al., 2006). In addition to undertaking longterm management of an episodic and often unpredictable disease, families must frequently manage multiple comorbidities including asthma, allergic rhinitis, and food and environmental allergies (Kapoor et al., 2008), which are all more common in children with $\mathrm{AD}$, and can make treatment more complex. Stigmatisation of individuals with $\mathrm{AD}$ has been reported, and appearance-related teasing and bullying can make an already frustrating and difficult condition even more distressing to manage (Magin et al., 2008).

Lack of adherence to $\mathrm{AD}$ management plans is common and presents a threat to successful management (Chisolm et al., 2009, Krejci-Manwaring et al., 2007). A study by Storm et al. (2008) found that $47.8 \%$ of prescriptions for children attending a dermatology outpatient clinic remained unfilled. Even where medications are supplied directly to parents and regular follow-up is provided, objectively-measured adherence rates are as low as 32\% (Krejci- 
Manwaring et al., 2007). Recent evidence suggests that apparent lack of response to traditional therapy, or failure of therapy after a good initial response, may be due to waning adherence to the $\mathrm{AD}$ management plan as opposed to increasing resistance to topical medications over time (Krejci-Manwaring et al., 2007).

Characteristics of children with $\mathrm{AD}$ and their parents have the potential to make management difficult. Children with $\mathrm{AD}$ tend to experience more emotional and behavioural problems than their healthy peers (Absolon et al., 1997, Dennis et al., 2006, Elliott and Luker, 1997, Pauli-Pott et al., 1999, Reichenberg and Broberg, 2004), and the presence of increased difficulties has been verified by independent observation of child behaviour as well as by parent report (e.g. Daud et al., 1993). A link between more profound emotional and behavioural problems and greater AD severity has been identified by some studies (Absolon et al., 1997, Daud et al., 1993) but not others (e.g. Dennis et al., 2006); these contradictory results may be explained by wide variations in age and AD severity of child samples, and in measures of $\mathrm{AD}$ severity and child behaviour. Elevated levels of parenting stress, depression, and anxiety are also more common among parents of children with AD (Daud et al., 1993, Faught et al., 2007, Pauli-Pott et al., 1999), and reports of marriage instability attributed to the strain of caring for a child with AD are frequent (Daud et al., 1993, Elliott and Luker, 1997, Lawson et al., 1998).

There is an emerging literature in the broader field of child chronic disease management suggesting that behaviour problems in children with chronic diseases may affect parents' ability to implement their child's treatment plan. Relationships have been found between child behaviour problems and difficulty managing children with asthma (Burgess et al., 2008, Chiang et al., 2003, Morawska et al., 2008) and children who have undergone renal transplantation (Gerson et al., 2004). It is also widely acknowledged that high levels of parenting stress or poorer psychological functioning may impair the ability of the parent to 
meet the needs of a child with a chronic health condition (Burgess et al., 2008, Gerson et al., 2004).

Health behaviour theories recognise that the social environment may impact health by influencing individuals' behaviour (Rimer and Glanz, 2005). Considering the bidirectional influences that exist between parent, child, and family may contribute to an understanding of factors that influence behaviour of parents when managing the health-related needs of a child with a chronic health condition. Thus, Bandura's (1977) theory of self-efficacy was selected as the conceptual framework for this study. The concept of self-efficacy was first proposed by Bandura in 1977, who defined efficacy expectation as "the conviction that one can successfully execute the behaviour required to produce the outcomes" (Bandura, 1977, p.193). Self-efficacy theory proposes that, given adequate skills and incentives, an individual's reaction to obstacles and adversity in a given situation, the amount of effort they will apply, and their level of perseverance, will be determined by their self-efficacy beliefs (Bandura, 1977, Bandura, 1997).

Self-efficacy beliefs have been found to have causal effects on human functioning and behaviour. However, numerous factors may impact on beliefs and thereby influence actual behaviour. An individual's self-efficacy beliefs can be bolstered by experiences of success at a particular task; vicarious experience of success, through observing behaviours being successfully modelled by others; or verbal persuasion of their ability to carry out behaviours despite past failures. Conversely, self-efficacy beliefs may be undermined by repeated failures. Individuals who are persuaded by others that they lack competence may avoid the task and give up when faced with adversity. Experiences of stress or low mood may also diminish self-efficacy beliefs, leading to avoidance of challenging situations, thus restricting the opportunity to develop coping skills and experience success (Bandura, 1995, Bandura, 1977). 
While the role of self-efficacy in adult chronic disease management has received attention in the literature, very few studies have examined psychosocial correlates of parents' selfefficacy when caring for a child with a chronic health condition. Available research, however, suggests that child behaviour difficulties (Heller, 1993), parenting stress related to caring for a child with a chronic illness (Streisand et al., 2005) and socioeconomic status (Grus et al., 2001) may all influence parents' self-efficacy for managing their child's chronic health condition. Self-efficacy in turn has been associated with variations in parents' performance of disease management tasks, such as asthma management behaviours (e.g. assessing symptoms, administering medication, monitoring) (Chiang et al., 2003) and cystic fibrosis management (monitoring and treating respiratory infections, obstructions, malabsorption, and malnutrition) (Bartholomew et al., 1993), for example. Perhaps most importantly, greater self-efficacy for managing the child's condition has been associated with indicators of reduced morbidity, such as fewer and less severe asthma symptoms, fewer missed school days, and fewer emergency department presentations (Bursch et al., 1999, Grus et al., 2001).

Psychological support and education of parents and caregivers is of key importance to successful AD management, and although measures of parents' self-efficacy have been suggested as useful indicators of the effectiveness of interventions (Ersser et al., 2007), published research examining relationships between parents' self-efficacy and performance of AD management tasks continues to be extremely limited (Ersser et al., 2014). Likewise, relationships between self-efficacy or task performance, and psychosocial characteristics of children and their parents, remain virtually unexplored in the literature.

The main aim of the study was to answer the following two research questions: (i) Do child, parent, and family characteristics influence (a) parents' self-efficacy for performing AD management tasks, and (b) parents' self-reported performance of $\mathrm{AD}$ management tasks? 
(ii) Does self-efficacy mediate relationships between child, parent, and family characteristics and parents' self-reported performance of AD management tasks?

The secondary aim of the study was to use direct observation of parent behaviour during a routine $\mathrm{AD}$ management session to examine relationships between child, parent, and family characteristics, parents' self-efficacy for managing the child's AD, and parents' observed treatment competence.

Lastly, it was hypothesised that parent-reported emotional and behavioural problems in children with severe $\mathrm{AD}$ would be more profound compared to children with mild or moderate AD.

\section{MATERIALS AND METHODS}

A cross-sectional study design was used. Parent-child dyads were recruited from dermatology clinics at a paediatric tertiary hospital in Brisbane, Australia, from September 2009 to June 2010. Inclusion criteria for children were as follows: aged 2-12 years with (a) a diagnosis of AD confirmed by dermatology registrar or consultant; (b) AD history of $\geq 3$ months; (c) no other chronic health condition other than $\mathrm{AD}$, asthma, allergic rhinitis, or allergy. For each child, one primary caregiving parent was asked to participate. Permission to conduct the research was granted by the Queensland University of Technology Human Research Ethics Committee, and by the Children's Health Services District Ethics Committee. Parents and (where able) children gave written consent to participate.

The main research questions required use of multiple regressions to test whether up to 13 continuous variables could explain variation in one continuous outcome variable. Assuming a large effect size $\left(f^{2}=0.35\right)$, a minimum 64 parent-child dyads were needed to provide $80 \%$ power with a type 1 error of 5\% (two-tailed) for the models to be tested (Cohen, 1992). 


\section{Parent-Report Questionnaire Measures}

The Child Eczema Management Questionnaire (CEMQ) (see Mitchell and Fraser, 2011, for details of reliability and validity) evaluates parents' self-efficacy, outcome expectations, and self-reported task performance when managing their child's AD. It contains three scales: (i) the modified Parent Self-Efficacy with Eczema Care Index (PASECI), which assesses parents' self-efficacy for managing various aspects of their child's AD; (ii) the Parent Eczema Management Scale (PEMS), used to assess parents' self-reported performance of AD management tasks; and (iii) the Parent Outcome Expectations of Eczema Management Scale (POEEMS), which assesses parents' expectations that performance of key management tasks would result in positive outcomes for their child's AD. Each scale comprises 25 items representing key $\mathrm{AD}$ management tasks, and respondents rate each item using 11-point Likert scales anchored at 0 and 10 (PASECI: cannot do at all - highly certain can do; PEMS: never - always (successful); POEEMS: not at all helpful-always helpful). Total scores for each scale range from 0-10, and higher scores indicate greater self-efficacy, more successful task performance, and more positive outcome expectations. Internal consistency was excellent for the present sample for PASECI $(\alpha=.95)$, PEMS $(\alpha=.94)$, and POEEMS $(\alpha=.96)$.

The Eyberg Child Behavior Inventory (ECBI) (Eyberg and Pincus, 1999) is a robust measure assessing parent-reported behaviour problems for children aged 2-16 years. Frequency of 36 behaviours are rated from 1 (never) to 7 (always) to generate an Intensity score. Behaviours considered problematic by the parent are identified (yes/no) to generate a Problem score. Intensity scores $\geq 131$ and Problem scores $\geq 15$ indicate significant behaviour problems. There was good internal consistency ( $\alpha=.95$ and .90 , respectively) for the present sample.

\section{The Strengths and Difficulties Questionnaire (SDQ) Emotional Symptoms subscale}

(Goodman, 1997) is used widely to screen for emotional problems in children over the 
previous six months. The Emotional Symptoms subscale comprises five items which are rated from 0 (not true) to 2 (certainly true) to generate the subscale score. It has demonstrated good reliability and validity (Mellor, 2005), and internal consistency was satisfactory for the present sample $(\alpha=.70)$.

The Depression Anxiety Stress Scales - 21 item version (DASS-21) (Lovibond and Lovibond, 1995) assesses symptoms of depression, anxiety, and stress in adults over the past week. It comprises Depression, Anxiety, and Stress scales, each of which contains 7 items rated from 0 (did not apply to me at all) to 3 (applied to me very much, or most of the time). Raw scale scores range from $0-21$, and are doubled so that they are comparable to those from the 42-item DASS. DASS-21 has demonstrated good discriminant and concurrent validity (Antony et al., 1998), and there was good internal consistency in the current sample for the Depression $(\alpha=.93)$, Anxiety $(\alpha=.81)$, and Stress $(\alpha=.93)$ scales.

The Parenting Stress Index-Short Form (PSI-SF) (Abidin, 1995) is a 36-item measure of global parenting stress. Total scores $>90$ indicate clinically significant levels of stress. The PSI-SF has demonstrated excellent concurrent validity (Abidin, 1995), and internal consistency was excellent for the present sample $(\alpha=.95)$.

The Quality Marriage Index (QMI) (Norton, 1983) is a six-item questionnaire used to measure relationship quality and satisfaction. It contains five items assessing different aspects of the couple relationship using a 7-point Likert scale response format. An additional item assesses overall happiness within the relationship on a scale of 1 to 10 . Scores range from 645 , with scores $<30$ indicative of relationship difficulties. Internal consistency was excellent for the present sample $(\alpha=.97)$.

The Parent Problem Checklist (PPC) (Dadds and Powell, 1991) is a 16-item questionnaire used to assess inter-parental conflict over child-rearing issues. The extent to which each issue has been a problem over the past 4 weeks is rated from 1 (not at all) to 7 (very much), to 
generate an Extent score. Issues considered to be a problem by the parent are identified (yes/no) to generate a Problem score. Problem scores range from 0-16, and scores $>5$ are considered to be in the clinical range, indicating the need for intervention. Internal consistency was good for the present sample for Extent ( $\alpha=.93)$ and Problem $(\alpha=.88)$ scores.

Socio-demographic Questionnaire. Socio-demographic items included child age and gender, respondent age and gender, relationship of the respondent to the child, parents' relationship status, number of siblings, parents' history of atopic disease, total number of hospitalisations for $\mathrm{AD}$, parents' number of years of formal education, and average annual family income.

\section{Observational Measures}

Scoring Atopic Dermatitis (SCORAD) was developed by the European Task Force on Atopic Dermatitis (1993) to create a consensus on AD severity assessment. It combines assessment of extent of lesions and intensity (erythema, oedema/papules, effect of scratching, oozing/crust formation, lichenification, and dryness), and subjective symptoms (itch and sleeplessness). Scores range from 0-103 $(<25=$ mild, $25-50=$ moderate, $>50=$ severe AD $)$. It is the only published $\mathrm{AD}$ severity measure to have demonstrated adequate content and construct validity, inter-rater reliability, and sensitivity to change (Schmitt et al., 2007).

Observation of a routine $\mathrm{AD}$ treatment session was conducted, and a global rating made for parents' competence in providing appropriate and adequate $\mathrm{AD}$ treatment based on the child's current clinical condition. Scores ranged from 1 (low competence) to 5 (high competence). A score of 5 (high competence) was given if topical corticosteroids and/or emollients were applied to appropriate areas, in appropriate amounts, and wet-wrap dressings were used if required. A score of 3 was made if treatment was generally appropriate, but topical medications were used in insufficient amounts, or an important treatment modality 
(e.g. wet wraps) was neglected to the extent that, while the child's condition was not likely to significantly deteriorate, neither was it likely to substantially improve. A score of 1 (low competence) was made if treatment was inappropriate or insufficient, to the extent that the child's condition was likely to deteriorate further.

\section{Data Collection}

Parents were provided with a questionnaire booklet at the time of recruitment into the study.

Home visits were scheduled for two weeks post-recruitment, and parents were asked to complete the booklet beforehand. At the home visits, questionnaire booklets were collected, visual assessments of AD severity conducted, and SCORAD scores calculated based on these assessments. Assessments were carried out independently by two registered nurses with expertise in paediatric $\mathrm{AD}$ management. Assessors were trained using the SCORAD Training Atlas, which is effective for standardising scoring of AD by personnel across different disciplines (Oranje et al., 1997). Both nurses independently assessed $20 \%$ of the sample, and inter-rater reliability was excellent (intra-class correlation $r=.99$ ). Bland-Altman analysis confirmed agreement, with differences lying within limits of agreement (mean difference \pm $2 S D$ ) for 12 out of 13 children assessed. When scores were categorised into severity classes (mild, moderate, severe), agreement was perfect $(\kappa=1.0)$.

Observation of a routine treatment session was conducted for children assessed as having moderate or severe AD based on their SCORAD score. Observations were conducted at home, timed to coincide with the child's usual treatment session, and conducted in the room where treatment was usually done. Video-recording started once the child's bath was complete, and continued for the duration of the skincare session (typically 5-20 minutes). Parents were asked to remain in the observation area, ensure that the child remained in the area once the session had commenced, and interact as naturally as possible. The researcher remained out of direct sight of parent and child (where possible) to minimise reactivity 
effects. Video-recordings of all the treatment sessions were reviewed independently by two registered nurses with expertise in paediatric AD management, who assessed parents' treatment competence. Inter-rater reliability was good (weighted $\kappa=.71)$, with perfect agreement for $62 \%$ of parents. Disagreements between assessors were $\leq 1$ point.

\section{Data Analysis}

Analyses were undertaken using SPSS 17.0. Descriptive statistics were used to summarise characteristics of the sample. Parametric statistical tests were used for normally distributed data, while non-parametric tests were used for data that were not normally distributed. Initially, general relationships between all key variables and demographic variables were assessed using Pearson's and Spearman's correlation coefficients. Between-groups ANOVAs and Kruskall-Wallis tests were used to examine differences between groups. A significance level of .05 was used. Less than $4 \%$ of data were missing overall, and the pattern of missing data was completely random.

Hierarchical multiple regressions examined relationships between (i) AD management self-efficacy, and (ii) self-reported performance of AD management tasks, and other child, parent, and family variables. Selection of variables for inclusion was guided by the conceptual framework underpinning the study and an initial evaluation of crude relationships between the variables. Where multiple variables assessed the same conceptual area, only that with the most complete data or the most precise level of measurement was included in the models. Models included parents reporting marriage or de facto relationships only, as the contexts in which single, separated, or divorced parents responded to questionnaires examining parenting conflict (PPC) or relationship satisfaction (QMI) were uncertain.

Finally, Baron and Kenny’s criteria and the Sobel test (Baron and Kenny, 1986), as well as the bootstrapping methodology described by Preacher and Hayes (2004), were used to test whether self-efficacy mediated relationships between child, parent, and family variables and 
parents' performance of AD management tasks. First, a series of regression models was used to test for mediation effects according to Baron and Kenny's (1986) criteria: namely, that a variable may be considered a mediator if (i) the independent variable/s are significant predictors of the presumed mediator; (ii) the mediator is a significant predictor of the dependent variable; and (iii) the mediator significantly predicts the dependent variable after controlling for the independent variable/s, diminishing previously significant relationships between the independent and dependent variables. Sobel tests were used to formally test statistical significance of the difference between total and direct effects (Baron and Kenny, 1986). The bootstrapping approach to mediation analysis was also used to test significance of indirect effects, as it improves power while limiting Type I error (MacKinnon et al., 2002), and makes no assumptions about the sampling distribution of the test statistic or the variables. It provides a point estimate of the indirect effect, and a 95\% confidence interval which indicates a statistically significant indirect effect at the .05 level if the confidence interval does not include zero (Preacher and Hayes, 2004).

\section{RESULTS}

\section{Sample}

Of the 90 parent-child dyads within the target age range who attended the clinic between September 2009 and June 2010, all met inclusion criteria and were invited to participate. Of these, 12 declined, 12 withdrew prior to data collection, and two children were ineligible as siblings were already enrolled. The final sample consisted of 64 parent-child dyads (participation rate $=72.73 \%$ ). Most parents $(90.6 \%, 58)$ were female, with a mean age of 35.70 years $(S D=7.97)$, and an average 13.06 years $(S D=2.81)$ formal education. Children were predominantly boys $(64.1 \%, 41)$, with a mean age of 5.88 years $(S D=3.21$, median $=5$ years, range 2-12). Median age at diagnosis was 4.5 months (birth-72 months), and 85.9\% 
(55) were diagnosed before one year of age. Almost half $(48.4 \%, 31)$ had at least one hospital separation for AD.

Families comprised a median two children (1-6) with a median annual household income of $\$ 50,000-\$ 75,000$ per year. Most parents $(81.3 \%, 52)$ were in a married or de facto relationship. Chi-squared goodness of fit tests confirmed representativeness of the sample in terms of family composition compared to 2006 Australian census population data (Australian Bureau of Statistics, 2007, Australian Bureau of Statistics, 2008), although the median income range for couple families was somewhat lower than the Australian average for couple families with dependent children (\$98,956 per year) (Australian Bureau of Statistics, 2009).

\section{Predicting self-efficacy and self-reported performance of AD management tasks}

Descriptive statistics for child, parent, and family variables are presented in Table 1.

Correlation coefficients for relationships among child, parent, and family variables and parents' self-efficacy and self-reported task performance are provided in Table 2.

Predicting parents' self-efficacy for performing AD management tasks. Hierarchical multiple regression was used to assess the ability of child, parent, and family variables to explain variation in parents' self-efficacy for performing AD management tasks (PASECI scores) (Table 3). Child emotional difficulty and parent anxiety were not included due to weak, non-significant correlations with the dependent variable. Parent education was selected for inclusion over household income because of a stronger correlation with the dependent variable. Parent stress (DASS scores) was selected for inclusion over parenting stress (PSI scores) due to high $(>.70)$ correlations between parenting stress and two other variables (global stress and depression). Four variables were transformed to reduce skewness, and improve normality, linearity and homoscedasticity of residuals: logarithmic transformations were used on DASS Depression and Stress scores, and PPC Intensity scores, while QMI scores were reflected and logarithmic transformation applied. Using a $p<.001$ criterion for 
Mahalanobis distance, no outliers were identified.

There was no relationship between self-efficacy and the set of sociodemographic variables entered at step $1, F(3,48)=1.99, p=.128$, although parents' education explained a significant proportion of variation. Addition of parent adjustment variables at step 2 contributed significantly to the prediction equation, $F_{\text {change }}(2,46)=4.64, p=.015$, and parents' education and stress both explained significant proportions of variance. Addition of family adjustment variables at step 3 also contributed significantly to prediction of self-efficacy, $F_{\text {change }}(2,44)=$ $4.09, p=.023$, and parents' education continued to explain a significant proportion of variation, although stress was no longer significant after parenting conflict and relationship satisfaction scores were included. Child variables added at step 4 made the most significant contribution to the model, $F_{\text {change }}(2,42)=7.29, p=.002$. Total variance explained by the model (adjusted $R^{2}$ ) was 43.7\%, $F(9,42)=5.41, p<.001$. Child behaviour difficulties ( $p=$ $.003)$ and parents' education $(p=.006)$ made statistically significant unique contributions to the final model. There was no substantial difference between the final model and models resulting from complete case analysis, and analysis without transformation of variables.

Predicting parents' self-reported performance of AD management tasks. A second hierarchical multiple regression was used to test whether the same variables could explain variation in parents' self-reported performance of AD management tasks (PEMS scores) (Table 4). There was no relationship between task performance and the set of sociodemographic variables entered at step $1, F(3,48)=1.76, p=.167$. Addition of parent adjustment variables at step 2 significantly contributed to the prediction equation, $F_{\text {change }}(2,46)=4.77, p=.013$, and parents' stress explained a significant proportion of variation in task performance. Addition of family adjustment variables at step 3 contributed significantly to prediction of task performance, $F_{\text {change }}(2,44)=4.09, p=.023$, although parents' relationship satisfaction was the only variable to explain a statistically significant 
proportion of unique variance; stress no longer made a significant contribution to the model. Finally, child variables added at step 4 made a significant contribution to the model, $F_{\text {change }}(2,42)=4.68, p=.015$. Total variance explained by the model (adjusted $R^{2}$ ) was $37.5 \%, F(9,42)=4.41, p<.001$. Parents' education $(p=.029)$ made the greatest significant unique contribution to the final model, followed by child behaviour $(p=.042)$. AD severity closely approached statistical significance $(p=.050)$. There was no substantial difference between the final model and the model resulting from complete case analysis. When data were analysed without transformation of variables, child behaviour was the only variable to explain a significant proportion of variation in task performance, although parents' education closely approached statistical significance $(p=.051)$.

Testing a mediation model. Standard multiple regression was used to test whether selfefficacy would mediate the relationships between parents' education and child behaviour and self-reported performance of AD management tasks. Having satisfied conditions (i) and (ii) of Baron and Kenny's criteria (1986), a third regression was conducted to test whether parents' education and child behaviour would still explain significant proportions of variation in task performance after adjusting for self-efficacy (see Supplementary Table 1).

Total variance explained by the model (adjusted $R^{2}$ ) was $90.3 \% F(10,41)=48.74, p<$ .001 . Self-efficacy accounted for $43.7 \%$ of unique variation in self-reported task performance. After adjusting for self-efficacy, previously significant independent variables (parents' education and child behaviour) were no longer statistically significant, and regression coefficients were markedly reduced, suggesting that self-efficacy mediated the relationships between task performance and both parents' education and child behaviour.

Sobel tests were conducted to test the hypothesis of no difference between total effects of education and child behaviour on task performance, and direct effects of each variable on task performance after controlling for self-efficacy. Results indicated that self-efficacy was a 
statistically significant mediator of the effects of education $(z=-2.41, p=.016)$ and child behaviour $(z=-4.87, p<.001)$ on task performance, and self-efficacy still mediated the effects of both education $(z=-2.84, p=.004)$ and child behaviour $(z=-.15, p=.006)$ on task performance after adjusting for covariates included in the regression model.

The mediation model was further tested using Preacher and Hayes' bootstrapping methodology (2004) for indirect effects based on 1000 bootstrap resamples. Results confirmed self-efficacy as a significant mediator of the effects of education (point estimate $=$ $-.18,95 \%$ CI -.31,-..05) and child behaviour (point estimate $=-.03,95 \%$ CI -.04,-..02) on task performance, and 95\% confidence intervals not including zero indicated statistically significant indirect effects at the .05 level (see Figure 1). Self-efficacy still mediated the effects of education (point estimate $=-.20,95 \% \mathrm{CI}-.33,-.08$ ) and child behaviour (point estimate $=-.02,95 \% \mathrm{CI}-.04, .01)$ after adjusting for covariates included in the regression model.

\section{Observation of AD treatment sessions}

Forty-three parent-child dyads were asked to participate in a treatment observation session, as the child's AD was assessed as being moderate or severe. Of these, 22 agreed to participate (participation rate $=51.2 \%$ ). Reasons cited by parents choosing not to participate included: the child was not receiving routine treatment for their $\mathrm{AD}(9.3 \%, 4)$; the child's treatment was not carried out or supervised by a parent $(14.0 \%, 6)$; time pressures $(11.6 \%, 5)$; child or family stress $(7.0 \%, 3)$; and living or travelling away from home $(7.0 \%, 3)$. Participants had a higher mean $\mathrm{AD}$ severity score $(M=54.90, S D=13.68)$ compared to non-participants $(M=$ 41.00, $S D=12.43), \mathrm{t}(40)=3.45, \mathrm{p}=.001$, and a higher mean score for child emotional symptoms $(U=187.5, z=-2.01, \mathrm{p}=.045)$; there were no significant differences between the groups on other key variables.

Over half of parents $(52.4 \%, 11)$ were assessed as demonstrating high competence (score 
of 5) with carrying out their child's treatment, while $14.3 \%(n=3)$ and $23.8 \%(n=5)$ of parents were scored 4 and 3 respectively. One parent was scored 2, and only one parent was assessed as having low competence, scoring 1 out of 5 . Five $(23.8 \%)$ children actively participated in their treatment, typically assisting with application of topical medication, or, in one case, applying creams and ointments under the parent's supervision. There was a negative relationship between participation in treatment and their age at diagnosis $\left(r_{p b}=-.49\right.$, $n=21, p=.026)$, but no relationship between participation and child age, duration of AD, number of hospital separations, or AD severity.

Correlations between parents' observed treatment competence and key variables are presented in Table 2. Importantly, there were positive relationships between observed treatment competence and parents' self-efficacy for managing AD, and self-reported success in performing $\mathrm{AD}$ management tasks. There was also a strong relationship between observed treatment competence and outcome expectations of task performance (rho $=.60, n=21, p=$ $.004)$.

\section{Child behavioural and emotional difficulties and AD severity}

A one-way between-groups ANOVA was used to test the hypothesis that behavioural problems (ECBI Intensity scores) were more profound for children with severe, compared to mild or moderate, AD. Five children lacked valid ECBI scores due to missing data, and were excluded from this analysis. Mean EBCI Intensity scores for children with mild $(n=20)$, moderate $(n=21)$, and severe $(n=18)$ AD were $98.75(S D=23.62), 97.86(S D=30.99)$, and $126.11(S D=39.09)$, respectively, and were significantly different across groups, $\mathrm{F}(2,56)=$ 4.863, $\mathrm{p}=.011$. Post-hoc comparisons (Tukey HSD) indicated that mean scores for children with severe $\mathrm{AD}$ were significantly different from children with mild $(p=.027)$ or moderate $(p$ $=.020) \mathrm{AD}$, while the mild and moderate groups were not significantly different $(p=.995)$. Of children with valid ECBI Intensity scores $(n=59), 38.8 \%$ of children with severe AD 
scored in the clinical range for behavioural difficulty, compared to $9.5 \%$ and $5.0 \%$ of children with moderate or mild $\mathrm{AD}$, respectively.

A Kruskall-Wallis test was used to test the hypothesis that parent-reported emotional difficulty (SDQ Emotional Symptoms scores) was also more profound for children with severe, compared to mild or moderate, AD. Median scores for mild $(n=21)$, moderate $(n=$ $22)$, and severe $(n=21)$ AD were $2(0-6), 1(0-7)$, and $3(0-9)$, respectively, and were significantly different across the groups, $\chi^{2}(2, n=64)=10.87, p=.004$. Post-hoc comparisons (Mann-Whitney U tests with Bonferroni correction, significance level $=.017$ ) indicated that average scores for children with severe AD were significantly different from children with moderate $\operatorname{AD}(U=105.5, z=-3.10, p=.002)$. There was no significant difference between children with mild compared to moderate $(U=179, z=-1.30, p=.194)$, or mild compared to severe $(U=133, z=-2.24, p=.025)$, AD. However, $38.1 \%$ of children with severe $\mathrm{AD}$ scored in the borderline or high range for emotional difficulty, compared to $18.2 \%$ and $19.0 \%$ of children with moderate or mild $\mathrm{AD}$, respectively.

For children with valid scores for both the ECBI and the SDQ Emotional Symptoms subscale $(n=59)$, a small proportion $(8.5 \%, n=5)$ scored outside the normal range on both measures; of these, four children had severe AD, and one had mild AD.

\section{Parents' education, self-efficacy, and child AD severity}

As displayed in Table 2, there was a negative correlation between parents' self-efficacy (PASECI) and AD severity (SCORAD), whereby greater self-efficacy for managing AD was associated with lower AD severity. Likewise, less severe AD was associated with more years of education reported by parents. However, examination of the full set of correlations (Table 2) revealed an unexpected relationship: despite negative correlations between self-efficacy and $\mathrm{AD}$ severity, and between $\mathrm{AD}$ severity and parents' education, parents' education was also, paradoxically, negatively correlated with self-efficacy. 
This pattern of results suggests a degree of heterogeneity within the sample. On the basis of the conceptual model underpinning the research, parents' education was considered the variable most likely to be exerting a moderating effect on relationships between the variables. Post-hoc analyses were therefore conducted to test the hypothesis that relationships between self-efficacy, education, and AD severity would be different for parents reporting more compared to fewer years of formal education. A median split categorised parent participants into lower ( $\leq 12$ years, $n=28)$ and higher ( $>12$ years, $n=36)$ education categories. For the lower education group, there were negative relationships between education and self-efficacy (rho $=-.42, n=28, p=.028$ ), and between self-efficacy and AD severity (rho $=-.38, n=28$, $p=.045$ ), but no relationship between education and $\mathrm{AD}$ severity (rho $=-.166, n=28, p=$ $.400)$. For the higher education group, there was no relationship between education and selfefficacy (rho $=-.05, n=36, p=.762$ ), and the relationship between self-efficacy and AD severity did not attain statistical significance (rho $=-.29, n=36, p=.085$ ). There was, however, a negative relationship between education and $\mathrm{AD}$ severity (rho $=.40, n=36, p=$ $.017)$.

\section{DISCUSSION}

This study examined factors with potential to influence parents' management of child AD, and makes progress toward addressing a gap in the literature that has hindered development of appropriate theory-based interventions to support improved management.

This appears to be one of the first studies to use validated measures of child behaviour and $\mathrm{AD}$ severity to examine relationships between these variables and parents' confidence and task performance when managing $\mathrm{AD}$ in children. Child behaviour problems, parental depression and stress, parenting conflict, and relationship dissatisfaction were all associated with lower self-efficacy and less self-reported success in performing AD management tasks. 
Of all of these variables, child behaviour was the most strongly correlated with both selfefficacy and task performance.

Each of these variables (child behaviour difficulties, parent self-efficacy, and performance of $\mathrm{AD}$ management tasks) were also correlated with $\mathrm{AD}$ severity. Child behaviour and parents' education explained significant proportions of variation in parents' self-efficacy and self-reported task performance, and self-efficacy emerged as a likely mediator for the relationships between both child behaviour and parent education, and self-reported performance of AD management tasks. These results should be interpreted with caution in light of a modest sample size and a strong relationship between the mediator (self-efficacy) and outcome (self-reported task performance) variables, which may have limited the capacity to detect significant relationships between both child behaviour and parents' education and parent-reported task performance after self-efficacy was included in the models. Nevertheless, the data appear to support the notion that, for child behaviour in particular, selfefficacy may mediate the relationship with parent-reported task performance. Even after adjusting for disease severity, parents of children with behavioural difficulties were at particular risk of low self-efficacy for managing the child's condition, and poorer selfreported and observed performance of $\mathrm{AD}$ management tasks.

These results build on findings from previous research which found that parents reported a lack of confidence with managing $\mathrm{AD}$ when their child was uncooperative with treatment (Mitchell and Fraser, 2014). Indeed, child resistance to treatment can be a significant problem for families of children with AD (Santer et al., 2013). Resistant behaviours, ranging from a simple lack of cooperation through to kicking and screaming during treatment, may prompt parents to adopt coercive parenting strategies (for example, the use of bribes or force), or to reduce the frequency of therapy, both of which may be counter-productive to successful longterm management and potentially damaging to the parent-child relationship (Santer et al., 
2013).

Observations of parents' competence when carrying out their child's treatment confirmed the relationship between parent-reported child behaviour difficulties and performance of AD management tasks. Although reasonably high levels of task performance were observed, with the majority of parents displaying high levels of treatment competence, there was substantial variation in the degree of skill demonstrated across the sample, and several parents provided treatment that was clearly inadequate given the severity of the child's clinical condition. Research using direct observational methods to assess the behaviour of parents and children engaged in disease management tasks is scarce, and this study appears to be one of the first to use behavioural observation to assess parents' performance of AD management tasks. This is significant, as previous research has relied on parents' self-report of task performance, or of indirect measures of adherence to the child's management plan. The approach taken in the current study to assess parents' competence with $\mathrm{AD}$ management is noteworthy, and is a first step toward developing more reliable methods of behavioural assessment for use in clinical research with this group.

Strong relationships emerged between parents' self-efficacy, outcome expectations, and self-reported performance of AD management tasks, and parents' observed competence when providing routine $\mathrm{AD}$ treatment for their child. These findings provide evidence of the concurrent validity of PEMS, POEEMS, and PASECI, as parents reporting greater selfefficacy and more successful task performance were indeed observed to be performing AD management tasks at a more competent level compared to parents with lower scores on these measures. Furthermore, higher scores for outcome expectations of performing management tasks were associated with more competent task performance. These relationships are congruent with the principles of self-efficacy theory, which guided the original development of these instruments (Mitchell and Fraser, 2011), and results therefore confirm that the 
instruments are valid and useful measures of their underlying theoretical constructs. Secondly, results provide a foundation for future research examining causal relationships between these variables. Although self-efficacy theory posits that self-efficacy and outcome expectation beliefs are consistently antecedent to an individual's behaviour, the crosssectional design used for this study precludes inferences about the direction of causal relationships between self-efficacy, outcome expectations, and self-reported and observed task performance. Longitudinal research is therefore needed to provide empirical evidence of temporal relationships between these variables. A greater understanding of the causal pathways between these variables will support the development of effective, evidence- and theory-based interventions to support parents in developing greater confidence and competence in caring for their child with AD.

Relationships between most variables were in the direction anticipated on the basis of selfefficacy theory. The negative relationship between parents' education and self-efficacy was unexpected, however: parents with more years of formal education tended to report lower self-efficacy for managing their child's condition. Yet, the children of parents with more years of formal education tended to have less severe AD. The relationship between parents' education and self-efficacy for managing $\mathrm{AD}$ could be explained in terms of a subset of "naively confident" parents, and this needs to be investigated in future research. Naively confident parenting (see, for example, Conrad et al., 1992, Hess et al., 2004) describes a subset of parents who report high levels of parenting self-efficacy, but at the same time feature low levels of parenting knowledge and parental behavioural competence (for example, lacking sensitivity to child's cues, poorly responsive to child's behaviour, and demonstrating fewer cognitive and social-emotional growth-fostering behaviours). In contrast, higher levels of parenting knowledge and greater self-efficacy together have been associated with greater parental behavioural competence. Applying this to the chronic illness 
context, there is potential for parent-focused interventions to lead to improved illness management and child health outcomes by incorporating intervention components to improve parents' knowledge about the disease and its management, as well as to improve their selfefficacy for managing their child's condition. Future research should therefore be concerned with examining relationships between parents' knowledge, self-efficacy beliefs, treatment competence, and child AD severity.

Participants in the current study were recruited sequentially from an outpatient clinic in a public hospital. Thus, conventional inferential statistics were employed to analyse data despite failure of the assumptions of random sampling and independence. Results should be considered generalizable primarily to children and families attending similar types of services.

\section{CONCLUSIONS}

In sum, results reveal the potential importance of child behaviour difficulties in the context of child $\mathrm{AD}$ management. Even after adjusting for $\mathrm{AD}$ severity and other child, parent, and family factors, child behaviour difficulties explained significant proportions of variation in parents' self-efficacy and self-reported performance of AD management tasks. Self-efficacy mediated the relationship between child behaviour and self-reported task performance. Observation of treatment sessions confirmed the relationship between child behaviour difficulties and parents' performance of management tasks. Thus, results indicate that parents of children with concurrent $\mathrm{AD}$ and behavioural difficulties are at risk of low self-efficacy for managing their child's condition, and less successful performance of management tasks. Of particular concern, results support the hypothesis that children with more profound behavioural difficulties are also likely to have more severe $\mathrm{AD}$, and thus represent a high-risk group of children whose parents may struggle to manage the disease successfully. While further longitudinal research is needed to elucidate the causal relationships between these 
variables, clinicians and researchers should consider the potential threat that child behaviour problems may pose to successful AD management. Future research will determine the best approach to address this issue, and will support the development of interventions to limit the impact of concurrent behavioural difficulties on the health and wellbeing of children with $\mathrm{AD}$, and their families.

\section{ACKNOWLEDGEMENTS}

The authors would like to express their sincere thanks to the parents and children who participated in this study.

Conflict of interest: None.

Ethics approval: Approval was granted by Queensland University of Technology (\#0900000654) and the Children's Health Services District Ethics Committee.

Funding: Funds granted through the Queensland Nursing Council Scholarship Scheme (Postgraduate Studies) enabled collection and analysis of observational data for this study. 


\section{REFERENCES}

Abidin, R.R., 1995. Parenting Stress Index (PSI) manual. Pediatric Psychology Press, Charlottesville, VA.

Absolon, C.M., Cottrell, D., Eldridge, S.M., Glover, M.T., 1997. Psychological disturbance in atopic eczema: the extent of the problem in school-aged children. British Journal of Dermatology 137 (2), 241-245.

Antony, M.M., Bieling, P.J., Cox, B.J., Enns, M.W., Swinson, R.P., 1998. Psychometric properties of the 42-item and 21-item versions of the Depression Anxiety Stress Scales in clinical groups and a community sample. Psychological Assessment 10 (2), 176-181.

Australian Bureau of Statistics, 2007. Census paints a picture of the average Australian family. ABS, Canberra.

Australian Bureau of Statistics, 2008. Family characteristics and transitions 2006-2007 (reissue), cat. no. 4442.0. ABS, Canberra.

Australian Bureau of Statistics, 2009. Household income and income distribution, Australia, 2007-2008, cat. no. 6523.0. ABS, Canberra.

Bandura, A., 1995. Exercise of personal and collective efficacy in changing societies. In: Bandura, A. (Ed.), Self-efficacy in changing societies. Cambridge University Press, Cambridge.

Bandura, A., 1977. Self-efficacy: Toward a unifying theory of behavioral change. Psychological Review 84 (2), 191-215.

Bandura, A., 1997. Self efficacy: the exercise of control. W.H. Freeman and Company, New York. 
Baron, R.M., Kenny, D.A., 1986. The moderator-mediator variable distinction in social psychological research: conceptual, strategic, and statistical considerations. Journal of Personality and Social Psychology 51 (6), 1173-1182.

Bartholomew, L.K., Parcel, G.S., Swank, P.R., Czyzewski, D.I., 1993. Measuring selfefficacy expectations for the self-management of cystic fibrosis. Chest 103 (5), 15241530.

Ben-Gashir, M.A., Seed, P.T., Hay, R.J., 2004. Predictors of atopic dermatitis severity over time. Journal Of The American Academy Of Dermatology 50, 349-356.

Burgess, S.W., Sly, P.D., Morawska, A., Devadason, S.G., 2008. Assessing adherence and factors associated with adherence in young children with asthma. Respirology 13 (4), $559-563$.

Bursch, B., Schwankovsky, L., Gilbert, J., Zeiger, R., 1999. Construction and validation of four childhood asthma self-management scales: parent barriers, child and parent selfefficacy, and parent belief in treatment efficacy. Journal of Asthma 36 (1), 115-128.

Chiang, L., Huang, J., Lu, C., 2003. Educational diagnosis of self-management behaviors of parents with asthmatic children by triangulation based on PRECEDE-PROCEED model in Taiwan. Patient Education and Counseling 49 (1), 19-25.

Chisolm, S.S., Taylor, S.L., Gryzwacz, J.G., O'Neill, J.L., Balrishnan, R.R., Feldman, S.R., 2009. Health behaviour models: a framework for studying adherence in children with atopic dermatitis. Clinical and Experimental Dermatology 35, 228-232.

Cohen, J., 1992. A power primer. Psychological Bulletin 112 (1), 155-159.

Conrad, B., Gross, D., Fogg, L., Ruchala, P., 1992. Maternal confidence, knowledge, and quality of mother-toddler interactions: a preliminary study. Infant Mental Health Journal 13 (4), 353-362. 
Dadds, M.R., Powell, M.B., 1991. The relationship of interparental conflict and global marital adjustment to aggression, anxiety and immaturity in agressive nonclinic children. Journal of Abnormal Child Psychology 19, 553-567.

Daud, L.R., Garralda, M.E., David, T.J., 1993. Psychosocial adjustment in preschool children with atopic eczema. Archives of Disease in Childhood 69, 670-676.

Dennis, H., Rostill, H., Reed, J., Gill, S., 2006. Factors promoting psychological adjustment to childhood atopic eczema. Journal of Child Health Care 10 (2), 126-139.

Elliott, B.E., Luker, K., 1997. The experiences of mothers caring for a child with severe atopic eczema. Journal of Clinical Nursing 6 (3), 241-247.

Ersser, S., Cowdell, F., Latter, S., Gardiner, E., Flohr, C., Thompson, A.R., Jackson, K., Farasat, H., Ware, F., Drury, A., 2014. Psychological and educational interventions for atopic eczema in children (Review). Cochrane Database of Systematic Reviews (1), Art. No.: CD004054. DOI:

004010.001002/14651858.CD14004054.pub14651853.

Ersser, S., Latter, S., Sibley, A., Satherley, P.A., Welbourne, S., 2007. Psychological and educational interventions for atopic eczema in children (Review). Cochrane Database of Systematic Reviews (3), Art. No.: CD004054. DOI: 004010.001002/14651858.CD14004054.pub14651852.

European Task Force on Atopic Dermatitis, 1993. Severity scoring of atopic dermatitis: the SCORAD Index (consensus report of the European Task Force on Atopic Dermatitis). Dermatology 186, 23-31.

Eyberg, S.M., Pincus, D., 1999. Eyberg Child Behavior Inventory and Sutter-Eyberg Student Behavior Inventory - Revised: Professional manual. Psychological Assessment Resources, Odessa, FL. 
Faught, J., Bierl, C., Barton, B., Kemp, A., 2007. Stress in mothers of young children with eczema. Archives of Disease in Childhood 92 (8), 683-686.

Gerson, A.C., Furth, S.L., Neu, A.M., Fivush, B.A., 2004. Assessing associations between medication adherence and potentially modifiable psychosocial variables in pediatric kidney transplant recipients and their families. Pediatric Transplantation 8 (6), 543550.

Goodman, R., 1997. The Strengths and Difficulties Questionnaire: a research note. Journal of Child Psychology and Psychiatry, and Allied Disciplines 38 (5), 581-586.

Grus, C.L., Lopez-Hernandez, C., Delamater, A., Appelgate, B., Brito, A., Wurm, G., Wanner, A., 2001. Parental self-efficacy and morbidity in pediatric asthma. Journal of Asthma 38 (1), 99-106.

Heller, T., 1993. Self-efficacy coping, active involvement, and caregiver well-being throughout the life course among families of persons with mental retardation. In: Turnbull, A.P., Patterson, J.M., Behr, S.K., Murphy, D.L., Marquis, J.G., BlueBanning, M.J. (Eds.), Cognitive coping, families, and disability. Paul H. Brookes Publishing Co., Inc, Baltimore.

Hess, C.R., Teti, D.M., Hussey-Gardner, B., 2004. Self-efficacy and parenting of high-risk infants: the moderating role of parent knowledge of infant development. Applied Developmental Psychology 25, 423-437.

Kapoor, R., Menon, C., Hoffstad, O., Bilker, W., Leclerc, P., Margolis, D.J., 2008. The prevalence of atopic triad in children with physician-confirmed atopic dermatitis. Journal of The American Academy of Dermatology 58 (1), 68-73.

Kay, J., Gawkrodger, D.J., Mortimer, M.J., Jaron, A.G., 1994. The prevalence of childhood atopic dermatitis in a general population. Journal of the American Academy of Dermatology 30 (1), 35-39. 
Krejci-Manwaring, J., Tusa, M.G., Carroll, C., Camacho, F., Kaur, M., Carr, D., Fleischer, A.B., Balkrishnan, R., Feldman, S.R., 2007. Stealth monitoring of adherence to topical medication: Adherence is very poor in children with atopic dermatitis. Journal Of The American Academy Of Dermatology 56, 211-216.

Lawson, V., Lewis-Jones, M.S., Finlay, A.Y., Reid, P., Owens, R.G., 1998. The family impact of childhood atopic dermatitis: the Dermatitis Family Impact questionnaire. British Journal of Dermatology 138 (1), 107-113.

Lewis-Jones, S., 2006. Quality of life and childhood atopic dermatitis: the misery of living with childhood eczema. International Journal of Clinical Practice 60 (8), 984-992.

Lovibond, S.H., Lovibond, P.F., 1995. Manual for the Depression Anxiety Stress Scales. Psychology Foundation, Sydney.

MacKinnon, D.P., Lockwood, C.M., Hoffman, J.M., West, S.G., Sheets, V., 2002. A comparison of methods to test mediation and other intervening variable effects. Psychological Methods 7 (1), 83-104.

Magin, P., Adams, J., Heading, G., Pond, D., Smith, W., 2008. Experiences of appearancerelated teasing and bullying in skin diseases and their psychological sequelae: results of a qualitative study. Scandinavian Journal of Caring Sciences 22, 430-436.

Mellor, D., 2005. Normative data for the Strengths and Difficulties Questionnaire in Australia. Australian Psychologist 40 (3), 215-222.

Mitchell, A.E., Fraser, J.A., 2014. Management of atopic dermatitis in children: Evaluation of parents' self-efficacy, outcome expectations, and self-reported task performance using the Child Eczema Management Questionnaire. Neonatal, Paediatric, and Child Health Nursing (in press). 
Mitchell, A.E., Fraser, J.A., 2011. Parents'self-efficacy, outcome expectations, and selfreported task performance when managing atopic dermatitis in children: instrument reliability and validity. International Journal of Nursing Studies 48 (2), 215-226.

Morawska, A., Stelzer, J., Burgess, S., 2008. Parenting asthmatic children: identification of parenting challenges. Journal of Asthma 45 (6), 465-472.

Norton, R., 1983. Measuring marital quality: a critical look at the dependent variable. Journal of Marriage and the Family 45 (1), 141-151.

Oranje, A.P., Stalder, J.F., Taïeb, A., Tasset, C., de Longueville, M., et al, 1997. Scoring of atopic dermatitis by SCORAD using a training atlas by investigators from different disciplines. . Pediatric Allergy And Immunology: Official Publication of the European Society of Pediatric Allergy and Immunology 8 (1), 28-34.

Pauli-Pott, U., Darui, A., Beckmann, D., 1999. Infants with atopic dermatitis: maternal hopelessness, child-rearing attitudes and perceived infant temperament. Psychotherapy and Psychosomatics 68 (1), 39-45.

Preacher, K.J., Hayes, A.F., 2004. SPSS and SAS procedures for estimating indirect effects in simple mediation models. Behavior Research Methods, Instruments, and Computers $36(4), 717-731$.

Reichenberg, K., Broberg, A.G., 2004. Emotional and behavioural problems in Swedish 7- to 9-year olds with asthma. Chronic Respiratory Disease 1 (4), 183-189.

Rimer, B.K., Glanz, K., 2005. Theory at a glance: A guide for health promotion practice. National Institutes of Health, Washington DC.

Santer, M., Burgess, H., Yardley, L., Ersser, S.J., Lewis-Jones, S., Muller, I., Hugh, C., Little, P., 2013. Managing childhood eczema: qualitative study exploring carers' experiences of barriers and facilitators to treatment adherence. Journal of Advanced Nursing 69 (11), 2493-2501. 
Schmitt, J., Langan, S., Williams, H.C., 2007. What are the best outcome measurements for atopic eczema? A systematic review. The Journal of Allergy and Clinical Immunology 120 (6), 1389-1398.

Storm, A., Andersen, S.E., Benfeldt, E., Serup, J., 2008. One in 3 prescriptions are never redeemed: Primary nonadherence in an outpatient clinic. Journal Of The American Academy Of Dermatology 59, 27-33.

Streisand, R., Swift, E., Wickmark, T., Chen, R., Holmes, C.S., 2005. Pediatric parenting stress among parents of children with type 1 diabetes: the role of self-efficacy, responsibility, and fear. Journal of Pediatric Psychology 30 (6), 513-521.

Su, J.C., Kemp, A.S., Varigos, G.A., Nolan, T.M., 1997. Atopic eczema: its impact on the family and financial cost. Archives of Disease in Childhood 76 (2), 159-162.

Williams, H., Robertson, C., Stewart, A., Ait-Khaled, N., Anabwani, G., Anderson, R., Asher, I., Beasley, R., Bjorksten, B., Burr, M., Clayton, T., Crane, J., Ellwood, P., Keil, U., Lai, C., Mallol, J., Martinez, F., Mitchell, E., Montefort, S., Pearce, N., Shah, J., Sibbald, B., Strachan, D., von Mutius, E., Weiland, S.K., 1999. Worldwide variations in the prevalence of symptoms of atopic eczema in the International Study of Asthma and Allergies in Childhood. Journal of Allergy and Clinical Immunology $103(1), 125-138$.

Williams, H., Stewart, A., von Mutius, E., Cookson, W., Anderson, H.R., 2008. Is eczema really on the increase worldwide? Journal of Allergy and Clinical Immunology 121 (4), 947-954.e915.

Williams, H., Strachan, D., 1998. The natural history of childhood eczema: observations from the British 1958 birth cohort study. British Journal of Dermatology 139, 834-839.

Zuberbier, T., Orlow, S.J., Paller, A.S., Taïeb, A., Allen, R., Hernanz-Hermosa, J.M., Ocampo-Candiani, J., Cox, M., Langeraar, J., Simon, J.C., 2006. Patient perspectives 
on the management of atopic dermatitis. Journal of Allergy and Clinical Immunology

$118(1), 226-232$. 
Table 1: Descriptive statistics for child, parent, and family variables

\begin{tabular}{|c|c|c|c|c|c|}
\hline Variable & $\alpha$ & Categories & $\%$ (no.) & Mean $(S D)$ & $\begin{array}{c}\text { Median } \\
(\min -\max )\end{array}$ \\
\hline \multicolumn{6}{|l|}{ Child AD Severity } \\
\hline \multirow[t]{3}{*}{ SCORAD } & & Mild & $32.8(21)$ & $38.78(19.10)$ & $36(0-81)$ \\
\hline & & Moderate & $34.4(22)$ & & \\
\hline & & Severe & $32.8(21)$ & & \\
\hline \multicolumn{6}{|l|}{ Child Behaviour Difficulties } \\
\hline \multirow[t]{3}{*}{ ECBI Intensity } & .95 & Normal & $76.6(49)$ & $106.78(33.60)$ & $106(36-206)$ \\
\hline & & High & $15.6(10)$ & & \\
\hline & & Not reported & $7.8 \quad(5)$ & & \\
\hline \multirow[t]{3}{*}{ ECBI Problems } & .90 & Normal & $75.0(48)$ & $8.07(6.91)$ & $8(0-26)$ \\
\hline & & High & $17.2(11)$ & & \\
\hline & & Not reported & $7.8(5)$ & & \\
\hline \multicolumn{6}{|l|}{ Child Emotional Difficulties } \\
\hline \multirow[t]{3}{*}{ SDQ Emotional Symptoms } & .70 & Normal & $75.0(48)$ & $2.59(2.27)$ & $2(0-9)$ \\
\hline & & Borderline & 4.7 (3) & & \\
\hline & & High & $20.3(13)$ & & \\
\hline \multicolumn{6}{|l|}{ Parent Management of $A D$} \\
\hline PASECI (Self-efficacy) & .95 & - & - & $7.90(1.48)$ & $7.90(3.48-9.92)$ \\
\hline POEEMS (Task performance) & .96 & - & - & $8.62(1.41)$ & $6.47(1.67-10.00)$ \\
\hline PEMS (Outcome expectations) & .94 & - & - & $7.42(1.51)$ & $7.26(3.88-9.92)$ \\
\hline \multicolumn{6}{|l|}{ Parent Depression } \\
\hline \multirow[t]{5}{*}{ DASS Depression } & .93 & Normal & $67.2(43)$ & $7.66(9.74)$ & $4(0-42)$ \\
\hline & & Mild & $15.6(10)$ & & \\
\hline & & Moderate & $6.3(4)$ & & \\
\hline & & Severe & 4.7 (3) & & \\
\hline & & Extremely severe & $6.3 \quad(4)$ & & \\
\hline \multicolumn{6}{|l|}{ Parent Anxiety } \\
\hline \multirow[t]{5}{*}{ DASS Anxiety } & .81 & Normal & $76.6(49)$ & $4.34(5.98)$ & $2(0-22)$ \\
\hline & & Mild & $6.3(4)$ & & \\
\hline & & Moderate & $6.3(4)$ & & \\
\hline & & Severe & $6.3(3)$ & & \\
\hline & & Extremely severe & $4.7 \quad(4)$ & & \\
\hline \multicolumn{6}{|l|}{ Parent Stress } \\
\hline \multirow[t]{5}{*}{ DASS Stress } & .93 & Normal & $71.9(46)$ & $11.50(11.18)$ & $8(0-42)$ \\
\hline & & Mild & $6.3(4)$ & & \\
\hline & & Moderate & $6.3(4)$ & & \\
\hline & & Severe & $7.8 \quad(5)$ & & \\
\hline & & Extremely severe & $7.8 \quad(5)$ & & \\
\hline \multicolumn{6}{|l|}{ Parenting Stress } \\
\hline \multirow[t]{4}{*}{ PSI Total Stress } & .95 & Normal & $57.8(37)$ & $77.75(25.06)$ & $78(36-137)$ \\
\hline & & High & $37.5(24)$ & & \\
\hline & & - clinical range & $36.1(22)$ & & \\
\hline & & Not reported & $4.7 \quad(3)$ & & \\
\hline \multicolumn{6}{|l|}{ Parenting Conflict } \\
\hline PPC Intensity & .93 & - & - & 36.43 (19.98) & $28.5(16-103)$ \\
\hline \multirow[t]{3}{*}{ PPC Problem } & .88 & Normal & $48.4(31)$ & $5.36(4.39)$ & $4(0-15)$ \\
\hline & & High & $39.1(25)$ & & \\
\hline & & Not reported & $12.5 \quad(8)$ & & \\
\hline
\end{tabular}




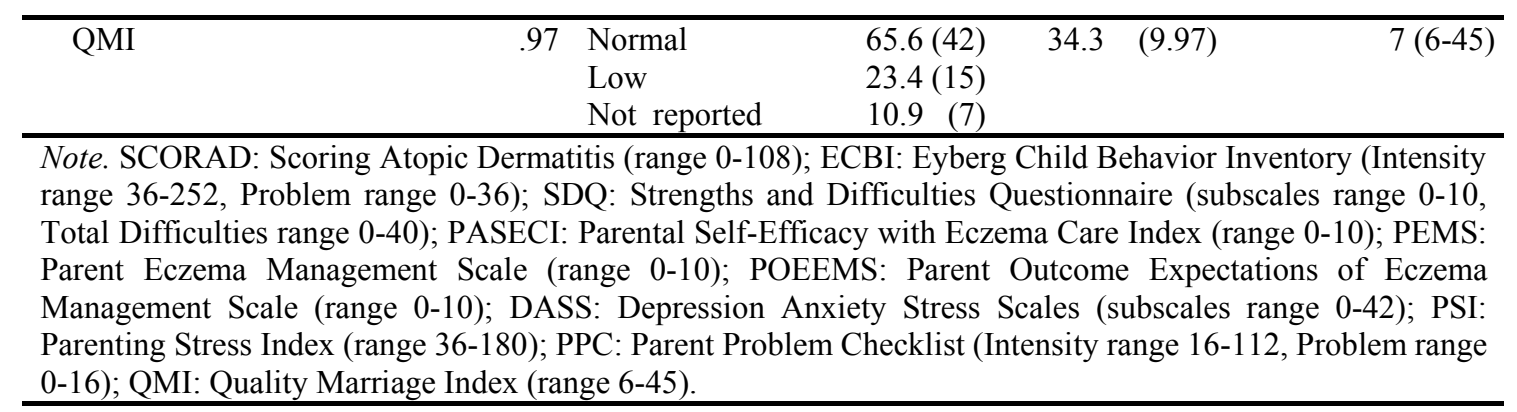


Table 2: Correlations among child, parent, and family variables, and parents' self-efficacy and performance of AD management tasks

\begin{tabular}{|c|c|c|c|c|c|c|c|c|c|c|c|c|c|c|c|c|}
\hline Variables & 1 & 2 & $3^{\mathrm{a}}$ & 4 & 5 & 6 & 7 & 8 & 9 & 10 & 11 & 12 & 13 & 14 & 15 & 16 \\
\hline 1. Self-efficacy & 1.00 & & & & & & & & & & & & & & & \\
\hline 2. Task performance & $.94^{* *}$ & 1.00 & & & & & & & & & & & & & & \\
\hline 3. Treatment competence ${ }^{\dagger a}$ & $.58^{* *}$ & $.47^{*}$ & 1.00 & & & & & & & & & & & & & \\
\hline 4. AD severity & $-.28^{*}$ & $-.35^{* *}$ & -.23 & 1.00 & & & & & & & & & & & & \\
\hline 5. Child behaviour & $-.46^{* *}$ & $-.42^{* *}$ & $-.47^{*}$ & $.38^{* *}$ & 1.00 & & & & & & & & & & & \\
\hline 6. Child emotional difficulty & -.11 & -.09 & -.33 & .23 & $.63^{* *}$ & 1.00 & & & & & & & & & & \\
\hline 7. Parent depression ${ }^{\dagger}$ & $-.32^{* *}$ & $-.26^{*}$ & $-.54^{*}$ & $.27^{*}$ & $.47^{* *}$ & $.36^{* *}$ & 1.00 & & & & & & & & & \\
\hline 8. Parent anxiety ${ }^{\dagger}$ & -.12 & -.04 & -.31 & .05 & .16 & .22 & $.46^{* *}$ & 1.00 & & & & & & & & \\
\hline 9. Parent stress ${ }^{\dagger}$ & $-.37^{* *}$ & $-.33^{* *}$ & $-.48^{*}$ & $.35^{* *}$ & $.48^{* *}$ & $.42^{* *}$ & $.60^{* *}$ & $.61^{* *}$ & 1.00 & & & & & & & \\
\hline 10. Parenting stress & $-.33^{* *}$ & $-.33^{* *}$ & $-.63^{* *}$ & $.35^{* *}$ & $.61^{* *}$ & $.54^{* *}$ & $.71^{* *}$ & $.40^{* *}$ & $.75^{* *}$ & 1.00 & & & & & & \\
\hline 11. Parenting conflict ${ }^{\dagger}$ & $-.32^{*}$ & $-.31^{*}$ & $-.58^{* *}$ & $.32^{*}$ & $.49^{* *}$ & .23 & $.54^{* *}$ & $.63^{* *}$ & $.66^{* *}$ & $.57^{* *}$ & 1.00 & & & & & \\
\hline 12. Relationship satisfaction ${ }^{\dagger}$ & $.34^{* *}$ & $.39^{* *}$ & $.49^{*}$ & -.18 & $-.41^{* *}$ & -.22 & $-.43^{* *}$ & $-.34^{* *}$ & $-.49^{* *}$ & $-.59^{* *}$ & $-.65^{* *}$ & 1.00 & & & & \\
\hline 13. Parent age & -.01 & -.01 & -.10 & -.05 & .01 & -.15 & .02 & .01 & .04 & -.02 & .12 & $-.34^{* *}$ & 1.00 & & & \\
\hline 14. Child age & .04 & .06 & -.01 & -.06 & $-.27^{*}$ & .04 & .09 & .04 & .04 & -.09 & .00 & -.18 & $.61^{* *}$ & 1.00 & & \\
\hline 15. Parents' education & $-.29^{*}$ & -.22 & -.13 & $-.28^{*}$ & .05 & $-.25^{*}$ & .07 & -.12 & .02 & -.08 & -.13 & .12 & -.01 & -.11 & 1.00 & \\
\hline 16. Household income ${ }^{\dagger}$ & $<-.01$ & -.04 & .22 & -.06 & -.04 & -.13 & -.07 & -.04 & -.13 & -.21 & -.25 & $.34^{*}$ & .09 & -.02 & $.25^{*}$ & 1.00 \\
\hline
\end{tabular}


Table 3: Hierarchical multiple regression predicting parents' self-efficacy for performing AD management tasks (PASECI)

\begin{tabular}{|c|c|c|c|c|c|c|}
\hline & $\beta$ & $95 \% \mathrm{CI}$ for $\beta$ & $t$ & $s r^{2}$ & $\begin{array}{l}\Delta R^{2} \\
\text { (adj) }\end{array}$ & $\begin{array}{c}\text { Cumulative } \\
R^{2} \text { (adj) }\end{array}$ \\
\hline \multicolumn{7}{|l|}{ Step 1} \\
\hline Parent age (years) & -.10 & $-.45, .24$ & -.60 & .006 & 055 & .055 \\
\hline Child age (years) & .04 & $-.32, .39$ & .21 & .001 & & \\
\hline Parent's education (years) & -.32 & $-.60,-.04$ & $-2.27^{*}$ & .095 & & \\
\hline \multicolumn{7}{|l|}{ Step 2} \\
\hline Parent age (years) & -.12 & $-.45, .21$ & -.75 & .009 & $.124^{*}$ & $.179^{* *}$ \\
\hline Child age (years) & -.01 & $-.35, .34$ & -.03 & $<.001$ & & \\
\hline Parent's education (years) & -.29 & $-.55,-.02$ & $-2.18^{*}$ & .077 & & \\
\hline Log DASS Stress & -.47 & $-.93,-.01$ & $-2.07^{*}$ & .069 & & \\
\hline Log DASS Depression & .10 & $-.36, .57$ & .45 & .003 & & \\
\hline \multicolumn{7}{|l|}{ Step 3} \\
\hline Parent age (years) & .03 & $-.30, .36$ & .20 & .001 & $.098^{*}$ & $.377^{* *}$ \\
\hline Child age (years) & -.08 & $-.41, .26$ & -.46 & .003 & & \\
\hline Parent's education (years) & -.33 & $-.59,-.07$ & $-2.54^{*}$ & .091 & & \\
\hline Log DASS Stress & -.32 & $-.80, .16$ & -1.34 & .026 & & \\
\hline Log DASS Depression & .22 & $-.22, .67$ & 1.01 & .014 & & \\
\hline Log PPC Intensity & -.13 & $-.53, .28$ & -.65 & .006 & & \\
\hline Reflect log QMI & -.34 & $-.68, .00$ & $-2.02^{\dagger}$ & .058 & & \\
\hline \multicolumn{7}{|l|}{ Step 4} \\
\hline Parent age (years) & .00 & $-.29, .29$ & .01 & $<.001$ & $.160^{* *}$ & $.437^{* *}$ \\
\hline Child age (years) & -.22 & $-.52, .09$ & -1.44 & .023 & & \\
\hline Parent's education (years) & -.35 & $-.60,-.11$ & $-2.89^{* *}$ & .092 & & \\
\hline Log DASS Stress & -.15 & $-.59, .29$ & -.67 & .005 & & \\
\hline Log DASS Depression & .21 & $-.19, .60$ & 1.05 & .012 & & \\
\hline Log PPC Intensity & -.10 & $-.46, .26$ & -.58 & .004 & & \\
\hline Reflect log QMI & -.19 & $-.50, .13$ & -1.20 & .016 & & \\
\hline SCORAD (AD severity) & -.21 & $-.45, .03$ & -1.76 & .034 & & \\
\hline ECBI Intensity & -.43 & $-.70,-.16$ & $-3.20^{* *}$ & .113 & & \\
\hline
\end{tabular}

Note. $n=52$. PASECI: Parental Self-Efficacy with Eczema Care Index; DASS: Depression Anxiety Stress Scales; PPC: Parent Problem Checklist; QMI: Quality Marriage Index; SCORAD: Scoring Atopic Dermatitis; ECBI: Eyberg Child Behavior Inventory.

${ }^{\dagger} p=.05 .{ }^{*} p<.05{ }^{* *} p<.01$. 
Table 4: Hierarchical multiple regression predicting parents' self-reported performance of AD management tasks (PEMS)

\begin{tabular}{lcccccc}
\hline & $\beta$ & $95 \%$ CI for $\beta$ & $t$ & & $\begin{array}{c}\Delta R^{2} \\
\text { (adj) }\end{array}$ & $\begin{array}{c}\text { Cumulative } \\
\Delta R^{2} \text { (adj) }\end{array}$ \\
\hline Step 1 & & & & & & \\
Parent age (years) & -.19 & $-.54, .16$ & -1.11 & -.10 & 043 & .043 \\
Child age (years) & .14 & $-.22, .49$ & .76 & .07 & & \\
Parent's education (years) & -.26 & $-.54, .03$ & -1.81 & -.28 & & \\
Step 2 & & & & & & \\
Parent age (years) & -.21 & $-.54, .13$ & -1.25 & -.10 & $.130^{*}$ & $.173^{*}$ \\
Child age (years) & .08 & $-.27, .43$ & .48 & .07 & & \\
Parent's education (years) & -.23 & $-.49, .04$ & -1.72 & -.28 & & \\
Log DASS Stress & -.51 & $-.97,-.05$ & $-2.23^{*}$ & -.40 & & \\
Log DASS Depression & .15 & $-.32, .61$ & .64 & -.27 & & \\
Step 3 & & & & & & \\
Parent age (years) & -.05 & $-.38, .28$ & -.32 & -.10 & $.098^{*}$ & $.271^{* *}$ \\
Child age (years) & .03 & $-.30, .36$ & .18 & .07 & & \\
Parent's education (years) & -.24 & $-.51, .02$ & -1.86 & -.28 & & \\
Log DASS Stress & -.42 & $-.90, .06$ & -1.77 & -.40 & & \\
Log DASS Depression & .27 & $-.17, .72$ & 1.24 & -.27 & & \\
Log PPC Intensity & .01 & $-.40, .41$ & .04 & -.37 & & \\
Reflect log QMI & -.42 & $-.76,-.07$ & $-2.44^{*}$ & -.49 & & \\
Step 4 & & & & & & \\
Parent age (years) & -.08 & $-.38, .23$ & -.51 & -.10 & $.104^{*}$ & $.375^{* *}$ \\
Child age (years) & -.08 & $-.40, .25$ & -.48 & .07 & & \\
Parent's education (years) & -.29 & $-.55,-.03$ & $-2.27^{*}$ & -.28 & & \\
Log DASS Stress & -.25 & $-.72, .21$ & -1.10 & -.40 & & \\
Log DASS Depression & .25 & $-.17, .66$ & 1.19 & -.27 & & \\
Log PPC Intensity & .02 & $-.36, .40$ & .09 & -.37 & & \\
Reflect log QMI & -.30 & $-.63, .04$ & -1.80 & -.49 & & \\
SCORAD (AD severity) & -.25 & $-.51, .00$ & $-2.10^{\dagger}$ & -.33 & & \\
ECBI Intensity & -.30 & $-.58,-.01$ & $-2.02^{*}$ & -.51 & & \\
& & & & & & \\
\hline
\end{tabular}

Note. $n=52$. PEMS: Parents' Eczema Management Scale; DASS: Depression Anxiety Stress Scales; PPC: Parent Problem Checklist; QMI: Quality Marriage Index; SCORAD: Scoring Atopic Dermatitis; ECBI: Eyberg Child Behavior Inventory.

${ }^{\dagger} p=.05 .{ }^{*} p<.05 .{ }^{* *} p<.01$. 
Supplementary Table 1: Standard multiple regression examining mediation by self-efficacy

\begin{tabular}{|c|c|c|c|c|}
\hline & $\beta$ & $95 \% \mathrm{CI}$ for $\beta$ & $\mathrm{t}$ & $\mathrm{sr}^{2}$ \\
\hline PASECI & .97 & $.84,1.10$ & $15.19^{* *}$ & 0.437 \\
\hline Parent age (years) & -.08 & $-.20, .04$ & -1.30 & 0.003 \\
\hline Child age (years) & .14 & $.01, .26$ & $2.11^{*}$ & 0.008 \\
\hline Parent's education (years) & .05 & $-.06, .16$ & 0.93 & 0.002 \\
\hline Log DASS Stress & -.11 & $-.29, .07$ & -1.20 & 0.003 \\
\hline Log DASS Depression & .05 & $-.12, .21$ & 0.55 & $<0.001$ \\
\hline Log PPC Intensity & .12 & $-.03, .27$ & 1.59 & 0.005 \\
\hline Reflect log QMI & -.11 & $-.25, .02$ & -1.74 & 0.006 \\
\hline SCORAD (AD severity) & -.05 & $-.15, .05$ & -0.96 & 0.002 \\
\hline ECBI Intensity & .12 & $-.01, .25$ & 1.94 & 0.007 \\
\hline \multicolumn{5}{|c|}{$\begin{array}{l}\text { Note. } n=52 \text {. Dependent variable: Parents' Eczema Management Scale scores. PASECI: Parental Self- } \\
\text { Efficacy with Eczema Care Index; DASS: Depression Anxiety Stress Scales; PPC: Parent Problem Checklist; } \\
\text { QMI: Quality Marriage Index; SCORAD: Scoring Atopic Dermatitis; ECBI: Eyberg Child Behavior } \\
\text { Inventory. } \\
{ }^{*} p<.05 .{ }^{* *} p<.01 \text {. }\end{array}$} \\
\hline
\end{tabular}



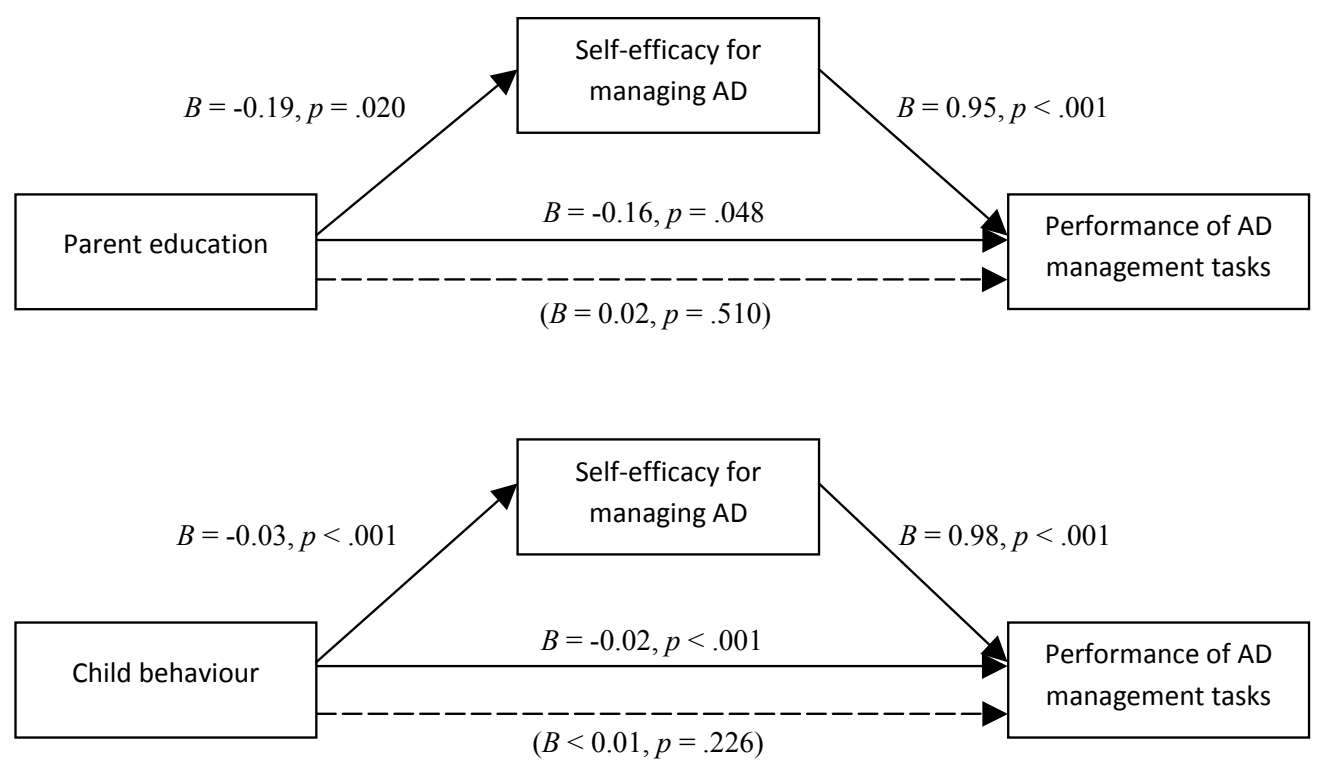

Figure 1: Mediation by self-efficacy. Figures represent unstandardised regression coefficients. Dashed arrows with coefficients in parentheses represent the direct effect of the independent variables (parent education, child behaviour) on performance of $\mathrm{AD}$ management tasks after controlling for self-efficacy. 


\section{What is already known about the topic?}

- Atopic dermatitis has a profound impact on the quality of life of affected children and their families, and parents are key to successful management.

- Child behaviour problems and parenting difficulties are more common in this clinical group, and have been linked to difficulties managing other chronic conditions in children.

\section{What this paper adds}

- Parents of children with concurrent atopic dermatitis and behaviour difficulties are at risk of lower self-efficacy for managing their child's condition, report less successful performance of management tasks, and demonstrate lower competence with providing routine treatment.

- Results suggest that parents' self-efficacy mediates the relationship between child behaviour difficulties and performance of management tasks.

- Interventions should focus on child behaviour and parenting issues to support parents of children with atopic dermatitis and improve child health outcomes. 